\title{
Original Research \\ ABCB1 limits the cytotoxic activity of TAK-243, an inhibitor of the ubiquitin-activating enzyme UBA1
}

\author{
Zhuoxun $\mathrm{Wu}^{1}$, Yuqi Yang ${ }^{1}$, Zining Lei ${ }^{1}$, Silpa Narayanan ${ }^{1}$, Jingquan Wang ${ }^{1}$, Qiuxu Teng $^{1}$, \\ Megumi Murakami ${ }^{2}$, Suresh V. Ambudkar ${ }^{2}$, Fengfeng Ping ${ }^{3, *}$, Zhesheng Chen ${ }^{1, *}$ \\ ${ }^{1}$ Department of Pharmaceutical Sciences, College of Pharmacy and Health Sciences, St. John's University, Queens, NY 11439, USA \\ ${ }^{2}$ Laboratory of Cell Biology, Center for Cancer Research, National Cancer Institute, NIH, Bethesda, MD 20892, USA \\ ${ }^{3}$ Department of Reproductive Medicine, Wuxi People's Hospital Affiliated to Nanjing Medical University, 214023 Wuxi, Jiangsu, China \\ *Correspondence: chenz@stjohns.edu (Zhesheng Chen); pingfengfeng2017@njmu.edu.cn (Fengfeng Ping) \\ Academic Editor: Graham Pawelec \\ Submitted: 25 September 2021 Revised: 20 November 2021 Accepted: 24 November 2021 Published: 5 January 2022
}

\begin{abstract}
Background: One of the major concerns of cancer therapy is the emergence of multidrug resistance (MDR). The MDR-associated ATPbinding cassette sub-family $\mathrm{B}$ member $1(\mathrm{ABCB} 1)$ transporter is established to mediate resistance against numerous anticancer drugs. In this study, we demonstrated that the Ubiquitin-like modifier activating enzyme 1 (UBA1) inhibitor TAK-243 is transported by the ABCB1. Methods: MTT assay was performed to evaluate the cytotoxicity of TAK-243. Western blot was carried out to investigate if TAK-243 affect to ABCB1 protein expression in cancer cells. High Performance Liquid Chromatography (HPLC) and ATPase assay were carried out to confirm TAK-243 as an ABCB1 substrate. $\left[{ }^{3} \mathrm{H}\right]$-paclitaxel accumulation assay was used to determine the MDR reversal effect of TAK-243. Computational docking analysis was performed to investigate the drug-transporter binding position. Results: The cytotoxicity profile showed that TAK-243 was less effective in ABCB1-overexpressing cells than in the parental cells, but pharmacological inhibition or knockout the gene of ABCB1 was able to reverse TAK-243 resistance. Furthermore, TAK-243 potently stimulated ABCB1 ATPase activity and the HPLC analysis revealed that TAK-243 accumulation was significantly reduced in ABCB1-overexpressing cells. Finally, the computational docking analysis indicates a high binding affinity between TAK-243 and human ABCB1 transporter. Conclusions: Our in vitro data characterized TAK-243 as a substrate of ABCB1, which may predict limited anticancer effect of this compound in drug resistant tumors.
\end{abstract}

Keywords: Ubiquitin-activating enzyme; TAK-243; Multidrug resistance; ABCB1; In vitro cytotoxicity

\section{Introduction}

ATP-binding cassette (ABC) transporters are membrane-bound transporters and some of the members of this superfamily play an important role in the energy-dependent efflux of substrate drugs [1]. The wellestablished $\mathrm{ABC}$ transporters responsible for multidrug resistance (MDR) are $\mathrm{ABCB} 1 / \mathrm{P}$-gp, $\mathrm{ABCG} 2 / \mathrm{BCRP}$, and $\mathrm{ABCC} 1 / \mathrm{MRP} 1$ [2], which are capable of protecting cancer cells from a wide range of structurally and mechanistically unrelated anticancer drugs. These ABC transporters may also affect to the pharmacokinetics and toxicities of clinically used anticancer drugs [3,4]. ABCB1 is the most studied $\mathrm{ABC}$ transporter, which was first discovered in 1976 [5]. ABCB1 extrudes neutral or positively charged hydrophobic chemicals and xenobiotics from the cells, serving as a protective mechanism in normal tissues [6]. However, studies on ABCB1 have revealed that chemotherapeutic agents such as taxanes, vinca alkaloids and anthracyclines as well as tyrosine kinase inhibitors (TKIs) such as dasatinib [7], ricolinostat [8] and GSK-1070916 are substrates of ABCB1 [9]. ABCG2 also has a diverse substrate profile, including mitoxantrone, irinotecan, methotrexate, doxorubicin, and TKIs [10,11].
ABCC1 was first identified from a doxorubicin-selected human small cell lung cancer cell line [12]. Compared with ABCB1, the substrates of ABCC1 are mostly amphipathic organic acids with more diverse structures including vinca alkaloids, anthracyclines and antiandrogens [13,14].

TKIs are small molecular drugs that target specific oncogenic tyrosine kinase to inhibit the proliferation, metastasis, invasion, and angiogenesis of cancer cells [15]. Recent studies have shown that some TKIs can reverse MDR by inhibiting the MDR-related ABC transporters [1619]. Most of the TKIs reverse MDR by downregulating the expression or blocking the efflux function of $\mathrm{ABC}$ transporters, which increase the amount of substrate drugs in cancer cells [20,21]. Therefore, repurposing TKIs as MDR modulators may be a potential strategy to combat MDR. However, it is worth noting that TKIs also are transported by $\mathrm{ABCB} 1$ or $\mathrm{ABCG} 2$, leading to suboptimal anticancer effect in drug resistant tumors [22-25].

TAK-243 is a first-in-class ubiquitin activating enzyme (UAE)/UBA1 inhibitor currently under clinical development [26,27]. The anticancer effect of TAK-243 is being evaluated in a clinical trial involving patients with leukemia (NCT03816319). Both UAE and UBA6 are 
known as E1 enzymes that regulate ubiquitin conjugation in mammals. UAE, encoded by $U B A 1$ gene, regulates the charging of over $99 \%$ of cellular ubiquitin while UBA6 charges less than $1 \%$ of cellular ubiquitin [28]. The protein ubiquitylation is triggered when UAE catalyzes ubiquitincharging for E2 enzymes, which in turns cooperate with cellular E3 ligases [29]. The clinical success of proteasome inhibitors gave rise to the development of smallmolecule inhibitors targeting the E1, E2, and E3 units. Several inhibitors are currently in clinical development, including pevonedistat, CGM097, avadomide, and indisulam [30]. Despite that the preclinical studies have established TAK-243 as a potent UBA1 inhibitor, drug resistance remains to be a major concern for targeted therapy. Several TKIs that target the ubiquitin pathway may interact with MDR-related ABC transporters. Besse et al. [31] showed that ABCB1 overexpression contributes to the reduced proteasome-inhibiting effect of carfilzomib but not bortezomib. MLN4924 was identified as an ABCG2 substrate in previous studies [23,32]. Lenalidomide is a weak substrate of $\mathrm{ABCB} 1$ but not $\mathrm{ABCG} 2$ or $\mathrm{ABCC} 1$ [33]. PYR41 was shown to prevent the reduction of $\mathrm{ABCB} 1$ protein expression and transport activity levels [34]. Therefore, it is critical to investigate the potential factors that predict TAK-243 sensitivity, which provides direction for the clinical usage. Yasuhisa et al. [35] confirmed that SLFN11 regulates the sensitivity of leukemia cells to TAK243. In SLFN11-KO cells, TAK-243 produced stronger protein ubiquitylation inhibition than in wide-type cells. Zhuang et al. [36] suggested that TAK-243 is more effective in bortezomib- and carfilzomib- resistant cell models. The significant cytotoxicity was also demonstrated in primary cells that acquired resistance to doxorubicin, melphalan, or dexamethasone.

While TAK-243 was demonstrated as a transported substrate of ABCG2 in previous studies [37,38], it is inconclusive whether ABCB1 or ABCC1 can interact with TAK243. Our results showed that the cytotoxicity of TAK-243 was attenuated in ABCB1-overexpressing cells but not in $\mathrm{ABCC} 1$-overexpressing cells, suggesting that it is a transported substrate of ABCB1.

\section{Materials and methods}

\subsection{Cell lines}

The parental cells and drug-resistant sublines overexpressing MDR transporters were used in this study, including human epidermoid carcinoma KB3-1, its ABCB1-overexpressing $\mathrm{KB}-\mathrm{C} 2$ subline and ABCC1-overexpressing KB-CV60 subline [39,40], human colon cancer SW620 and its ABCB1-overexpressing SW620/Ad300 subline [41]. HEK293 with stable pcDNA3.1- and ABCB1-transfected cell lines were included. ABCB1 knockout cell lines SW620-ABCB1ko and SW620/Ad300-ABCB1ko were established by CRISPR/CRISPR-associated (Cas) 9 system (unpublished data). All media were supplemented with $10 \%$ of fetal bovine serum (FBS) and 1\% of penicillin-streptomycin.

\subsection{Cellular toxicity assay}

MTT assay was conducted in accordance with the previous study [42]. Briefly, cells were harvested, counted, and seeded at $5 \times 10^{3}$ cells/well in 96-well plates for 24 h. Then cells were treated with different concentrations of the sample and further incubated for $72 \mathrm{~h}$. After that, MTT solution was added and incubated for $4 \mathrm{~h}$. Lastly, the solution was removed and DMSO was added to dissolve the formazan. $\mathrm{OD}_{750}$ value was read on an AccuSkan ${ }^{\mathrm{TM}} \mathrm{GO}$ UV/Vis Microplate Spectrophotometer (Thermo Fisher Scientific Inc., Waltham, MA, USA).

\subsection{Membrane ATPase assay}

The effect of TAK-243 on the vanadate-sensitive ATPase activity of ABCB1 in cell membrane prepared from High-Five insect cells was measured using the protocol as previous described [43]. Tepotinib was used as a reference ABCB1 ATPase inhibitor to evaluate the combination effect on ABCB1 ATPase activity [16].

\subsection{Determination of $A B C B 1$ protein expression level}

ABCB1-overexpressing KB-C2 cells were incubated with TAK-243 for up to 10 days. The total soluble protein lysates were prepared, subjected to SDS-PAGE, and transferred to PVDF membranes [44]. To quantify the expression level of $\mathrm{ABCB} 1$, membranes were probed with anti-ABCB1 monoclonal antibody (C219) (MA1-26528, Thermo Fisher Scientific Inc., Waltham, MA, USA). The loading control GAPDH was probed by anti-GAPDH monoclonal antibody (GA1R) (MA5-15738-D680, Thermo Fisher Scientific Inc., Waltham, MA, USA). Mouse IgG HRP conjugates (\#7076, Cell Signaling Technology Inc., Danvers, MA, USA) were used as secondary antibody. To visualize the bands, ECL detection reagents were used (Thermo Fisher Scientific Inc., Waltham, MA, USA). The protein band densitometry was measured using Image J software (NIH, MD, USA).

\subsection{Quantitative measurement of intracellular TAK-243 by} HPLC

The sample preparation and HPLC setting follows the previous mentioned protocol with modifications [38]. Cells were harvested, counted, and separated into microcentrifuge tubes $\left(1 \times 10^{6}\right.$ cell/tubes $)$ and incubated with 20 $\mu \mathrm{M}$ of TAK-243 with or without verapamil for $2 \mathrm{~h}$. Subsequently, samples were collected in $0.5 \%$ SDS with acetonitrile and centrifuged at $14,000 \mathrm{rpm}$ for $10 \mathrm{~min}$. The supernatants were collected, purified and subjected to HPLC analysis. 


\section{$2.6\left[{ }^{3} \mathrm{H}\right]$-paclitaxel accumulation assay}

The interaction of TAK-243 and ABCB1 substrate drug paclitaxel was determined by $\left[{ }^{3} \mathrm{H}\right]$-paclitaxel accumulation assay [45]. Briefly, cells were seeded into 24well plates overnight. Then, cells were incubated with plain medium or medium containing TAK-243 or Verapamil. Subsequently, $5 \mathrm{nM}$ of $\left[{ }^{3} \mathrm{H}\right]$-paclitaxel was added to each well and incubated for $2 \mathrm{~h}$. At the end, the radioactivity of samples was quantified by a liquid scintillation analyzer (Packard Instrument, Downers Grove, IL, USA).

\subsection{Computational drug-protein docking analysis}

The three-dimensional structure of TAK-243 was constructed to perform docking simulation with a human ABCB1 model 6QEX (obtained from RCSB Protein Data Bank) as previously described [46,47]. Docking calculations were performed using AutoDock Vina (version 1.1.2, Scripps Research, San Diego, CA, USA) and AutoDockTools (ADT, version 1.5.4, Scripps Research, San Diego, CA, USA) [48]. The top-scoring pose was selected for data analysis and visualization.

\subsection{Statistical analysis}

Data are collected from three independent experiments and presented as mean $\pm \mathrm{SD}$. One-way ANOVA was selected for the experimental analysis. The statistical analysis was performed in GraphPad Prism 8.1 (GraphPad Software, Inc, San Diego, CA, USA). A $p$ value below 0.05 was considered to be statistically significant.

\section{Results}

3.1 The cytotoxic effect of TAK-243 was attenuated in $A B C B 1$-overexpressing cells but not ABCC1-overexpressing cells

To characterize the interaction between TAK-243 and $\mathrm{ABCB} 1$ or ABCC1 transporters, MTT assay was performed to generate the cytotoxicity profiles (presented in Fig. 1) and the calculated $\mathrm{IC}_{50}$ values are summarized in Table 1. Compared to the parental KB-3-1 cells, ABCB1overexpressing KB-C2 cells demonstrated a 37.45-fold resistance to TAK-243 while ABCC1-overexpressing KBCV60 cells maintained sensitivity to same level as the parental KB-3-1 cells. We further confirmed these results in additional two pairs of ABCB1-overexpressing cell lines. HEK293/ABCB1 cells showed 10.62-fold resistance to TAK-243 compared to the parental HEK293/pcDNA3.1 cells. Similarly, drug-resistant SW620/Ad300 cells were highly (28.46-fold) resistant to TAK-243 compared to parental SW620 cells. Therefore, our results suggest that overexpression of $\mathrm{ABCB} 1$ but not $\mathrm{ABCC} 1$ affects the cytotoxic effect of TAK-243 in cancer cells.
3.2 Pharmacological inhibition and knock-out of $A B C B 1$ restore the sensitivity of MDR cells to TAK-243

Since overexpression of ABCB1 resulted in resistance to TAK-243, reversal studies were conducted to evaluate whether inhibiting $\mathrm{ABCB} 1$ can restore the cytotoxicity of TAK-243 in MDR cells. Verapamil, a known substrate of $\mathrm{ABCB} 1$ when used as a competitive inhibitor, was able to significantly enhances the cytotoxic effect of TAK-243 in ABCB1-overexpressing cells (Fig. 1A,C). When TAK-243 was co-incubated with verapamil, the foldresistance to TAK-243 significantly decreased from 37.45 to 3.15 -fold in $\mathrm{KB}-\mathrm{C} 2$ cells, and from 10.62- to 1.88 -fold in HEK293/ABCB1 cells. Furthermore, the cytotoxicity of TAK-243 was not affected in parental cells when combined with verapamil. The ABCC1 inhibitor MK-571 did not enhance TAK-243 cytotoxicity in parental and drug-resistant cells (Fig. 1B), suggesting TAK-243 is not a substrate of ABCC1. As shown in Fig. 1D, knockout of $A B C B 1$ gene also restored the sensitivity of SW620/Ad300 cell to TAK243. Furthermore, verapamil did not change the $\mathrm{IC}_{50}$ values of TAK-243 in ABCB1 knockout cells, suggesting that the sensitization effect is due solely to ABCB1 inhibition.

\subsection{TAK-243 stimulated the ABCB1 ATPase activity}

The interaction between ABCB1 and TAK-243 was evaluated in High-Five insect cell total membrane vesicles containing the ABCB1 transporter. As shown in Fig. 2A, TAK-243 in a concentration-dependent manner increased the ATP hydrolysis mediated by ABCB1. The ABCB1 ATPase activity reached a maximum of $181.3 \%$ stimulation when incubated with $20 \mu \mathrm{M}$ of TAK-243. To further verify that TAK-243 specifically stimulates ABCB1 ATPase activity, tepotinib was included as an ABCB1 ATPase inhibitor. When the membrane vesicles were incubated with both TAK-243 and tepotinib, the stimulatory effect of TAK-243 was significantly diminished (stimulation was decreased from $81.3 \%$ to $20.8 \%$ ). This result shows that TAK-243 can specifically stimulate ABCB1 ATPase activity. The cytotoxicity and ATPase data strongly indicate that it is a substrate of $\mathrm{ABCB} 1$ transporter.

\subsection{Overexpression of $A B C B 1$ reduced the intracellular accumulation of TAK-243}

As an MDR transporter, ABCB1 protects cancer cells by extruding substrate drugs into extracellular matrix. To this end, we measured the intracellular TAK-243 accumulation in empty vector transfectant HEK293/pcDNA3.1 cells and gene transfectant HEK293/ABCB1 and HEK293/ABCC1 cell lines. While drug-selected cancer cells may acquire other drug resistance mechanisms, the gene-transfected cells ensured that overexpression of $\mathrm{ABC}$ transporters is the sole contributor to MDR in HEK/ABCB1 and HEK293/ABCC1 cells. The cells were incubated with TAK-243 for $2 \mathrm{~h}$, followed by HPLC analysis. Our results demonstrated that the in- 

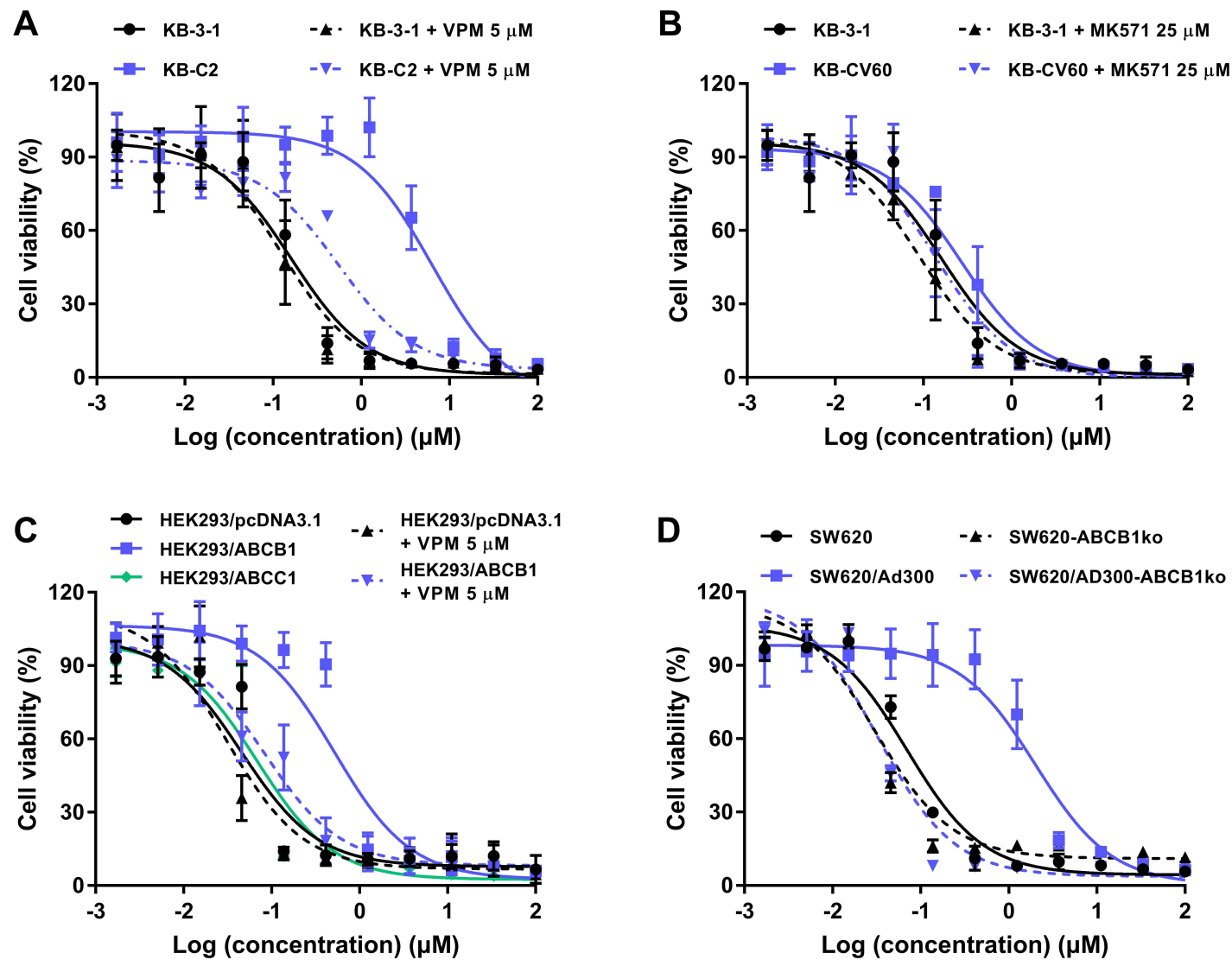

Fig. 1. The cytotoxic profile of TAK-243 in parental and drug-resistant cell lines. Cell viability curves for (A) KB-3-1 and KBC2 cells, (B) KB-3-1 and KB-CV60 cells, (C) HEK293/pcDNA3.1, HEK293/ABCB1, and HEK293/ABCC1 cells, and (D) SW620, SW620/Ad300, SW620-ABCB1ko, and SW620/Ad300-ABCB1ko cells. Verapamil and MK571 were used as inhibitor of ABCB1 and $\mathrm{ABCC} 1$, respectively. Data are expressed as mean $\pm \mathrm{SD}$ from three independent experiments.

A

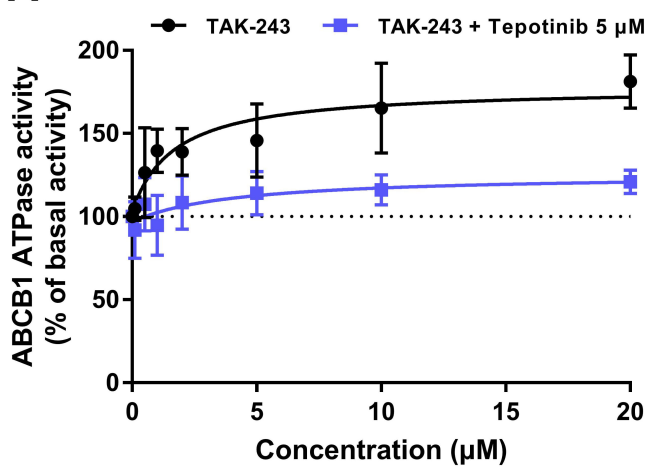

B

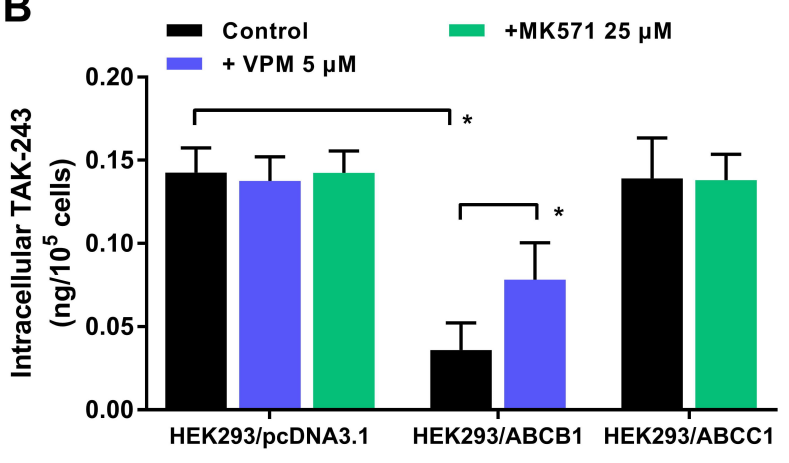

Fig. 2. TAK-243 stimulated the ABCB1 ATPase activity and was accumulated significantly less in ABCB1-overexpressing cells. (A) The effect of TAK-243 on ABCB1-mediated ATPase activity at concentration range from $0-20 \mu \mathrm{M}$. Tepotinib $(5 \mu \mathrm{M})$ was used as an ABCB1 ATPase inhibitor. (B) The intracellular concentration of TAK-243 in HEK293/pcDNA3.1, HEK293/ABCB1 and HEK293/ABCC1 cells. Verapamil is an ABCB1 inhibitor and MK571 is an ABCC1 inhibitor. Data are expressed as mean \pm SD derived from three independent experiments. ${ }^{*} p<0.05$ versus the control group.

tracellular concentration of TAK-243 was 3.7-fold lower in HEK293/ABCB1 than in HEK293/pcDNA3.1 cells. In addition, verapamil was able to significantly increase TAK243 concentration in HEK293/ABCB1 cells but not in the
HEK293/pcDNA3.1 cells. In contrast, the TAK-243 concentrations were similar between HEK293/pcDNA3.1 and HEK293/ABCC1 cells, and ABCC1 inhibitor MK571 did not affect the drug accumulation. Therefore, it suggests that 
Table 1. The $\mathrm{IC}_{50}$ of TAK-243 in ABCB1-overexpressing cells.

\begin{tabular}{|c|c|c|}
\hline \multirow{2}{*}{ Treatment } & \multicolumn{2}{|c|}{ IC_50 value $\pm \mathrm{SD}^{a}\left(\mathrm{mM}\right.$, Resistance fold $\left.{ }^{b}\right)$} \\
\hline & TAK-243 & TAK-243+ VPM $5 \mathrm{mM}$ \\
\hline KB-3-1 & $0.163 \pm 0.043(1.00)$ & $0.137 \pm 0.045(0.84)$ \\
\hline KB-C2 & $6.096 \pm 0.580(37.45)^{*}$ & $0.514 \pm 0.228(3.15)^{*}$ \\
\hline SW620 & $0.070 \pm 0.006(1.00)$ & $0.064 \pm 0.015(0.92)$ \\
\hline SW620/Ad300 & $1.991 \pm 0.225(28.46)^{*}$ & $0.065 \pm 0.023(0.93)$ \\
\hline SW620-ABCB1ko & $0.030 \pm 0.002(1.00)$ & $0.036 \pm 0.002(1.19)$ \\
\hline SW620/Ad300-ABCB1ko & $0.035 \pm 0.001(1.14)$ & $0.034 \pm 0.002(1.11)$ \\
\hline HEK293/pcDNA3.1 & $0.042 \pm 0.016(1.00)$ & $0.032 \pm 0.006(0.77)$ \\
\hline HEK293/ABCB1 & $0.441 \pm 0.130(10.62)^{*}$ & $0.079 \pm 0.026(1.88)$ \\
\hline $\begin{array}{l}{ }^{a} \mathrm{IC}_{50} \text { values are represent } \\
\text { iments. } \\
{ }^{b} \text { Rf: Resistance fold was } \\
243 \text { in drug-resistant cells } \\
{ }^{*} p<0.05 \text { versus the contr }\end{array}$ & $\begin{array}{l}\text { as mean } \pm \text { SD from thre } \\
\text { lculated by dividing the } \mathrm{I} \\
\text { the } \mathrm{IC}_{50} \text { of TAK- } 243 \text { in }\end{array}$ & $\begin{array}{l}\text { independent exper- } \\
\text { arental cells. }\end{array}$ \\
\hline
\end{tabular}

\begin{abstract}
ABCB1 is able to pump out TAK-243 from ABCB1 overexpressing cells, and this process can be inhibited by verapamil.
\end{abstract}

\subsection{TAK-243 did not upregulate ABCB1 expression level}

Some ABCB1 substrates have been shown to upregulate the protein expression level of $\mathrm{ABCB} 1$ transporter, therefore Western blot was performed to evaluate $\mathrm{ABCB} 1$ expression level after TAK-243 treatment. $\mathrm{ABCB} 1$-overexpressing cancer cells $\mathrm{KB}-\mathrm{C} 2$ were incubated with $1 \mu \mathrm{M}$ of TAK-243 for 10 consecutive days. As shown in Fig. 3A, the ABCB1 protein level remained constant during the incubation period. Therefore, TAK-243 may not affect the expression of ABCB1 in cancer cells.

\subsection{TAK-243 did not competitively inhibit the efflux of paclitaxel mediated by $A B C B 1$}

To determine whether TAK-243 can competitively inhibit ABCB1 efflux activity, $\left[{ }^{3} \mathrm{H}\right]$-paclitaxel accumulation assay was carried out using KB-3-1 and KB-C2 cells. As shown in Fig. 3B, compared to KB-3-1 cells, the accumulation of $\left[{ }^{3} \mathrm{H}\right]$-paclitaxel was 10 -fold lower in KB-C2 cells, indicating the active efflux function of $\mathrm{ABCB} 1$. Verapamil, a substrate of $\mathrm{ABCB} 1$ by competing for the binding site, inhibited the efflux and, significantly restored the paclitaxel accumulation in KB-C2 cells to the same level as that in KB-3-1 cells. However, TAK-243 did not demonstrate any effect on the efflux of paclitaxel in KB-C2 cells with concentrations around $\mathrm{IC}_{50}$ and $\mathrm{IC}_{85}$. Therefore, the results suggest that TAK-243 may not affect paclitaxel efflux by ABCB1.

\subsection{Docking of TAK-243 in the drug-binding pocket of human $A B C B 1$}

Since we identified TAK-243 as a substrate of $\mathrm{ABCB} 1$, protein-ligand docking was carried out to understand the potential binding pattern of TAK-243 with human ABCB1. Our results showed that the TAK-243 bound to $\mathrm{ABCB} 1$ with a $-8.023 \mathrm{kcal} / \mathrm{mol}$ affinity score. The ligandreceptor interactions are highlighted in Fig. 4B,C. TAK243 was stabilized in the ABCB1 substrate-binding site by both polar and hydrophobic interactions. Specifically, the pyrazolo-pyrimidine group in TAK-243 was stabilized by $\pi-\pi$ stacking with Trp232, and the sulfamate group was stabilized by multiple hydrogen bonds formed with $G \ln 195$, Gln347 and Glu875, respectively

\section{Discussion}

In this study, we performed in vitro assays to determine the influence of two MDR-related $\mathrm{ABC}$ transporters $\mathrm{ABCB} 1$ and $\mathrm{ABCC} 1$ on the cytotoxic effect of TAK-243 in cancer cells. TAK-243 is a first-in-class inhibitor of the ubiquitin-activating enzyme UBA1 to induce cancer cell death. TAK-243 was demonstrated to be effective against solid tumors including colon cancer and breast cancer in preclinical study, which distinguished it from proteasome inhibitors such as bortezomib and carfilzomib $[26,49]$. Many clinically used anticancer drugs were identified as substrates of MDR-related $\mathrm{ABC}$ transporters. These $\mathrm{ABC}$ transporters are drug efflux transporters that decrease the accumulation of anticancer drugs in cancer cells and therefore attenuate their cytotoxic effect. Clinical data have suggested that expression of $\mathrm{ABCB} 1$ and $\mathrm{ABCG} 2$ significantly affects the response of chronic myeloid leukemia (CML) patients to nilotinib and imatinib [50-52]. The clinical significance of $\mathrm{ABCC} 1$ was also demonstrated in many 

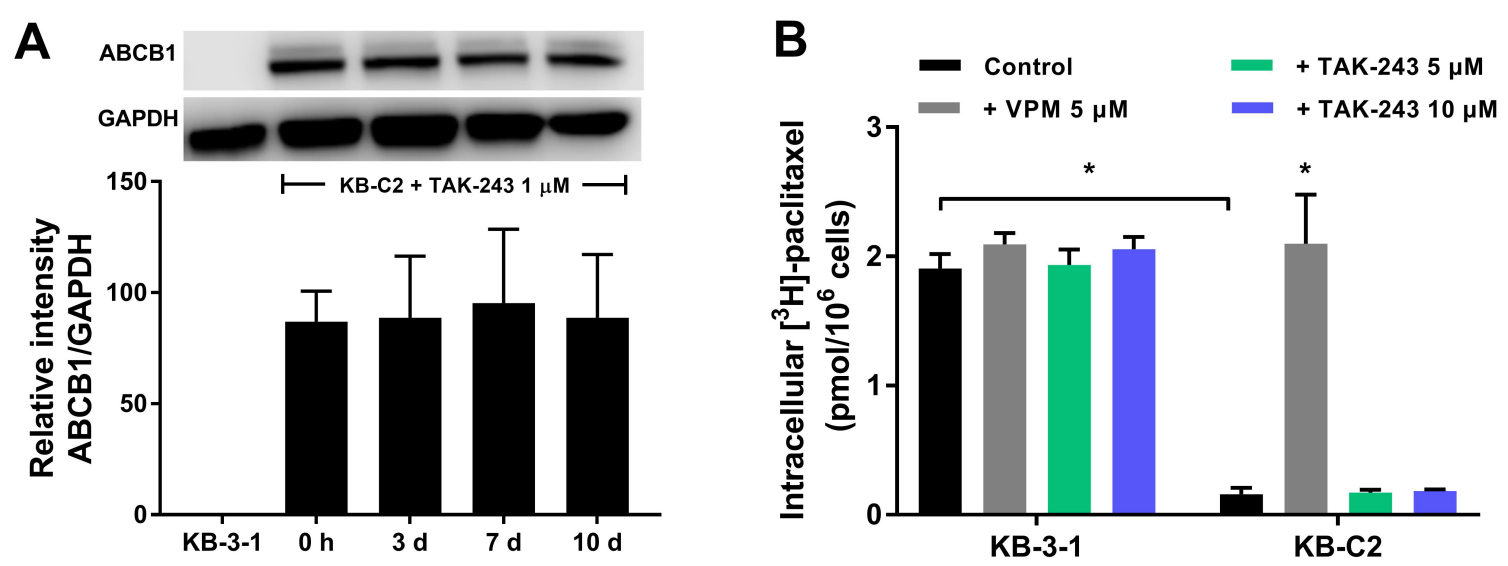

Fig. 3. TAK-243 did not affect ABCB1 transporter protein expression level, and did not inhibit paclitaxel efflux. (A) The effect of TAK-243 on ABCB1 expression level in KB-C2 cells after 10 days treatment. (B) The intracellular accumulation of [ $\left.{ }^{3} \mathrm{H}\right]$-paclitaxel in KB-3-1 and KB-C2 cells after co-incubated with 5 or $10 \mu \mathrm{M}$ of TAK-243. Verapamil was used as an ABCB1 inhibitor. Data are expressed as mean \pm SD derived from three independent experiments. ${ }^{*} p<0.05$ versus the control group.

studies. High expression of $\mathrm{ABCC} 1$ could predict poor response to chemotherapy or induce drug resistance in patients with breast cancer, ovarian cancer, and prostate cancer [53]. Therefore, it is critical to determine the interaction between TAK-243 and the MDR-related ABC transporters, which provides future direction for the development of a drug that can be used in the clinic. Evidence shown that ABCG2 actively extrudes TAK-243 and confers drug resistance in cancer cells [37,38].

The most significant finding of our study was that the cytotoxic effect of TAK-243 was inhibited in ABCB1but not $\mathrm{ABCC} 1$-overexpressing cells. The survival curves and $\mathrm{IC}_{50}$ values of TAK-243 were same between ABCC1overexpressing $\mathrm{KB}-\mathrm{CV} 60$ cells and the parental cells. In contrast, the $\mathrm{IC}_{50}$ values of $\mathrm{TAK}-243$ were significantly higher in $\mathrm{ABCB} 1$-overexpressing $\mathrm{KB}-\mathrm{C} 2$ and SW620/Ad300 cells compared to the parental KB-3-1 and SW620 cells, respectively, suggesting the overexpression of ABCB1 may contribute to TAK-243 resistance. Because the MDR cancer cells may employ multiple drug resistance mechanisms, both $A B C B 1$ gene transfected and $A B C B 1$ gene knockout cell models were used to further validate our finding. In gene transfected HEK293/ABCB1 cells, the cytotoxicity of TAK-243 was decreased as well. Furthermore, inhibition of $\mathrm{ABCB} 1$ function by gene knockout or $\mathrm{ABCB} 1$ substrate verapamil were able to sensitize MDR cancer cells to TAK-243. While in parental cells, ABCB1 knockout or verapamil did not enhance the cytotoxicity of TAK-243, suggesting that TAK-243 is a transported substrate of ABCB1.

To further characterize the interaction of TAK-243 with ABCB1, the ATPase activity in the presence of TAK243 was measured. The ABCB1 efflux function is driven by the energy derived from ATP-binding and hydrolysis, which is catalyzed by its nucleotide-binding domains
(NBDs). Our results showed that TAK-243 potently stimulated the ATPase activity of ABCB1. Furthermore, the co-incubation with ABCB1 ATPase inhibitor tepotinib decreased the stimulated ATPase activity, suggesting TAK243 is an $\mathrm{ABCB} 1$ substrate. Another important evidence is that the accumulation of TAK-243 was significantly decreased in ABCB1-overexpressing cells. After $2 \mathrm{~h}$ incubation with $20 \mu \mathrm{M}$ of TAK-243, only $30 \%$ of the drug remained in the HEK293/ABCB1 cells as compared to the HEK293/pcDNA3.1 cells. In addition, verapamil increased the intracellular level of TAK-243 in HEK293/ABCB1 cells but the effect was limited, which may be due to the insufficient incubation time of verapamil with the drug-resistant cells. Together, our results confirmed that TAK-243 is a transported substrate of $\mathrm{ABCB} 1$ and the cytotoxicity may be attenuated because of decreased TAK-243 accumulation in MDR cells.

Subsequently, two potential interactions between substrate and ABCB1 were explored. Some substrate drugs, such as paclitaxel and imatinib, are able to upregulate the expression of ABCB1 [51,54]. Our Western blot results showed that incubating $\mathrm{ABCB} 1$-overexpressing cells with TAK-243 for consecutive 10 days did not change the expression of ABCB1, suggesting that TAK-243 is unlikely to induce $\mathrm{ABCB} 1$ expression level. However, the effect of higher concentration of TAK-243 or longer incubation of TAK-243 should be further evaluated in parental and drug resistant cells. Another potential interaction is the competitive inhibition of ABCB1 efflux activity. Substrate drugs like gefitinib and nilotinib are able to modulate the efflux function of $\mathrm{ABCB} 1$ and reverse $\mathrm{ABCB} 1$-mediated drug resistance $[55,56]$. To this end, $\left[{ }^{3} \mathrm{H}\right]$-paclitaxel accumulation assay was performed to determine whether TAK-243 can inhibit drug efflux activity of ABCB1. Direct determination of $\left[{ }^{3} \mathrm{H}\right]$-paclitaxel showed that the intracellular pacli- 

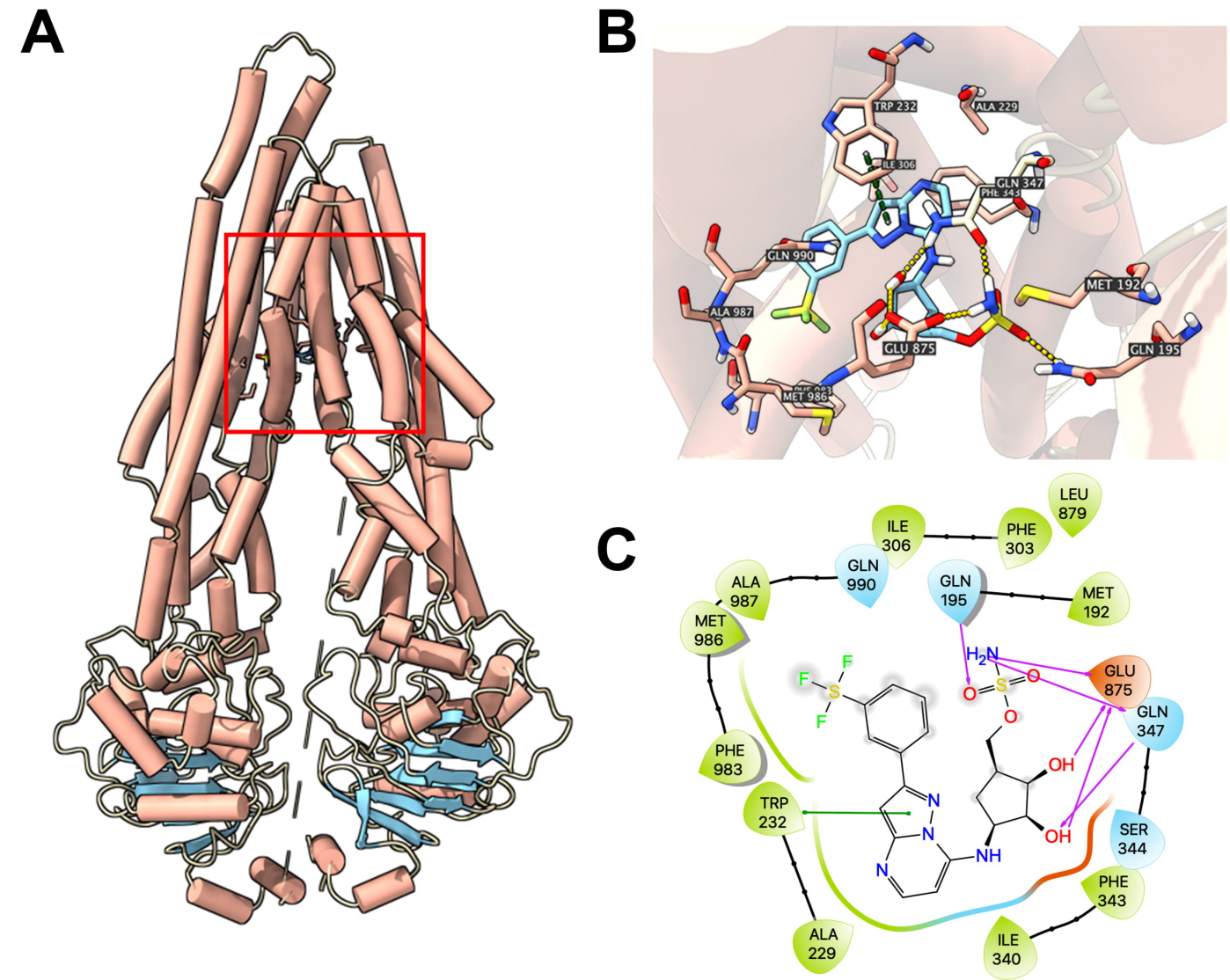

Fig. 4. Docking of TAK-243 in the drug-binding pocket of human ABCB1 protein model. (A) Overview of the best-scoring pose of TAK-243 in the drug-binding pocket of ABCB1 protein (6QEX). ABCB1 was displayed as colored tubes (helix: red; strand: blue; coil: white). TAK-243 was displayed as colored sticks. Carbon: cyan; oxygen: red; nitrogen: blue, hydrogen: white. (B) Details of the interaction between TAK-243 and ABCB1 binding pocket. ABCB1 helices were displayed as colored tubes (helix: red; strand: blue; coil: white). Important residues were displayed as colored sticks (carbon: red; oxygen: red; nitrogen: blue; hydrogen: white). TAK-243 was displayed as colored sticks (same as in (A). Hydrogen bonds were displayed as yellow dash lines. $\pi-\pi$ stacking interactions were displayed as green dash lines. (C) 2D diagram of the interaction between TAK-243 and ABCB1 binding pocket. Important amino acids within $3 \AA$ from the ligand were displayed as colored bubbles (green: hydrophobic; blue: polar). Purple solid lines with arrow indicate hydrogen bonds. Green solid lines indicate $\pi-\pi$ stacking interactions.

taxel concentration was significantly lower in KB-C2 cells compared to the KB-3-1 cells. While verapamil was able to inhibit paclitaxel efflux and completely restore paclitaxel accumulation in drug-resistant cells, TAK-243 failed to demonstrate any inhibition effect on the accumulation of paclitaxel. Therefore, our data suggests that TAK-243 does not serve as a reversal inhibitor of ABCB1. However, recent study has shown that $\mathrm{ABCB} 1$ may have multiple substrate-binding sites in the transmembrane domains that allows the binding of different substrates [57]. It is possible that TAK-243 may limit the efflux of some ABCB1 substrates that have the overlapping binding sites.

Lastly, the docking simulation analysis was carried out to predict the binding mode of TAK-243 with ABCB1. The computational analysis has been applied to the screening of substrate drugs with high accuracy, though the predicted interaction may not represent the actual binding mode [58]. The actual binding position should be further evaluated by cryogenic electron microscopy. The docking analysis showed that TAK-243 can form chemical bonds with Trp232, Gln195, Gln347 and Glu875 in the ABCB1 substrate-binding pocket with a high affinity score.

\section{Conclusions}

In summary, here we have shown that the ABCB1 actively transported TAK-243 and limited its cytotoxic effect in MDR cancer cells. Using an ABCB1 inhibitor or knockout of $A B C B 1$ gene was able to significantly abolish the TAK-243 resistance in MDR cancer cells. Thus, further in vivo studies with the animal models are necessary to validate these results. Therefore, if the results can be validated in vivo, it suggests that TAK-243 should be used in combination with an ABCB1 inhibitor in patients with MDR tumors to achieve optimal therapeutic effect. 


\section{Author contributions}

Conceptualization-ZXW,

FFP, $\quad$ ZSC; methodology-ZXW, YQY, JQW, ZNL, QXT, SN; resources-MM, SVA; writing-original draft preparationZXW; writing-review and editing - ZXW, FFP, SVA, ZSC; supervision-FFP and ZSC. All authors have read and agreed to the published version of the manuscript.

\section{Ethics approval and consent to participate}

Not applicable.

\section{Acknowledgment}

The authors would like to thank Shin-Ichi Akiyama (Kagoshima University, Kagoshima, Japan), and Susan E. Bates, Robert W. Robey (NCI, NIH, Bethesda, 331 Maryland, MD, USA) for kindly providing the cell lines. The authors would also like to thank ChemieTek (Indianapolis, IN) for providing the TAK-243.

\section{Funding}

This study was funded by the National Natural Science Foundation of China (81874330); Wuxi Taihu Lake Talent Plan Top Talents Project (BJ 2020001). The work was also partially supported by Department of Pharmaceutical Sciences, St. John's University. MM and SVA were supported by the Intramural Research Program, National Institutes of Health, National Cancer Institute, Center for Cancer Research.

\section{Conflict of interest}

The authors declare no conflict of interest. ZSC is serving as one of the Guest editors of this journal. We declare that ZSC had no involvement in the peer review of this article and has no access to information regarding its peer review. Full responsibility for the editorial process for this article was delegated to GP.

\section{References}

[1] Robey RW, Pluchino KM, Hall MD, Fojo AT, Bates SE, Gottesman MM. Revisiting the role of $\mathrm{ABC}$ transporters in multidrugresistant cancer. Nature Reviews Cancer. 2018; 18: 452-464.

[2] To KKW, Poon DC, Wei Y, Wang F, Lin G, Fu L. Pelitinib (EKB-569) targets the up-regulation of $\mathrm{ABCB} 1$ and $\mathrm{ABCG} 2$ induced by hyperthermia to eradicate lung cancer. British Journal of Pharmacology. 2015; 172: 4089-4106.

[3] Szakács G, Váradi A, Özvegy-Laczka C, Sarkadi B. The role of $\mathrm{ABC}$ transporters in drug absorption, distribution, metabolism, excretion and toxicity (ADME-Tox). Drug Discovery Today. 2008; 13: 379-393.

[4] Hegedűs C, Özvegy-Laczka C, Apáti A, Magócsi M, Német K, Örfi L, et al. Interaction of nilotinib, dasatinib and bosutinib with $\mathrm{ABCB} 1$ and $\mathrm{ABCG}$ : implications for altered anti-cancer effects and pharmacological properties. British Journal of Pharmacology. 2009; 158: 1153-1164.

[5] Juliano RL, Ling V. A surface glycoprotein modulating drug permeability in Chinese hamster ovary cell mutants. Biochimica Et Biophysica Acta. 1976; 455: 152-162.
[6] Sharom FJ. The P-glycoprotein multidrug transporter. Essays in Biochemistry. 2011; 50: 161-178.

[7] Chen Y, Agarwal S, Shaik NM, Chen C, Yang Z, Elmquist WF. P-glycoprotein and breast cancer resistance protein influence brain distribution of dasatinib. The Journal of Pharmacology and Experimental Therapeutics. 2009; 330: 956-963.

[8] Wu C, Hsieh Y, Murakami M, Vahedi S, Hsiao S, Yeh N, et al. Human ATP-binding cassette transporters ABCB1 and ABCG2 confer resistance to histone deacetylase 6 inhibitor ricolinostat (ACY-1215) in cancer cell lines. Biochemical Pharmacology. 2018; 155: 316-325.

[9] Wu ZX, Yang Y, Wang JQ, Zhou WM, Chen J, Fu YG, et al. Elevated ABCB1 Expression Confers Acquired Resistance to $\mathrm{Au}-$ rora Kinase Inhibitor GSK-1070916 in Cancer Cells. Frontiers in Pharmacology. 2020; 11: 615824.

[10] Mao Q, Unadkat JD. Role of the breast cancer resistance protein (BCRP/ABCG2) in drug transport-an update. The AAPS Journal. 2015 ; 17: 65-82.

[11] Hegedüs C, Truta-Feles K, Antalffy G, Várady G, Német K, Ozvegy-Laczka C, et al. Interaction of the EGFR inhibitors gefitinib, vandetanib, pelitinib and neratinib with the ABCG2 multidrug transporter: implications for the emergence and reversal of cancer drug resistance. Biochemical Pharmacology. 2012; 84: 260-267.

[12] Cole SP, Bhardwaj G, Gerlach JH, Mackie JE, Grant CE, Almquist KC, et al. Overexpression of a transporter gene in a multidrug-resistant human lung cancer cell line. Science. 1992; 258: $1650-1654$

[13] Kumar A, Jaitak V. Natural products as multidrug resistance modulators in cancer. European Journal of Medicinal Chemistry. 2019; 176: 268-291.

[14] Deeley RG, Cole SPC. Substrate recognition and transport by multidrug resistance protein 1 (ABCC1). FEBS Letters. 2006; 580: 1103-1111.

[15] Baudino T. Targeted Cancer Therapy: the next Generation of Cancer Treatment. Current Drug Discovery Technologies. 2015; 12: 3-20.

[16] Wu Z, Teng Q, Cai C, Wang J, Lei Z, Yang Y, et al. Tepotinib reverses ABCB1-mediated multidrug resistance in cancer cells. Biochemical Pharmacology. 2019; 166: 120-127.

[17] Wang J, Wang JQ, Cai CY, Cui Q, Yang Y, Wu ZX, et al. Reversal Effect of ALK Inhibitor NVP-TAE684 on ABCG2Overexpressing Cancer Cells. Frontiers in Oncology. 2020; 10: 228.

[18] Wu Z, Yang Y, Wang G, Wang J, Teng Q, Sun L, et al. Dual TTK/CLK2 inhibitor, CC-671, selectively antagonizes ABCG2mediated multidrug resistance in lung cancer cells. Cancer Science. 2020; 111: 2872-2882.

[19] Gao HL, Gupta P, Cui Q, Ashar YV, Wu ZX, Zeng L, et al. Sapitinib Reverses Anticancer Drug Resistance in Colon Cancer Cells Overexpressing the ABCB1 Transporter. Frontiers in Oncology. 2020; 10: 574861.

[20] Yang Y, Ji N, Cai CY, Wang JQ, Lei ZN, Teng QX, et al. Modulating the function of $\mathrm{ABCB} 1$ : in vitro and in vivo characterization of sitravatinib, a tyrosine kinase inhibitor. Cancer Communications. 2020; 40: 285-300.

[21] Wang T, Dong J, Yuan X, Wen H, Wu L, Liu J, et al. A New Chalcone Derivative C49 Reverses Doxorubicin Resistance in MCF7/DOX Cells by Inhibiting P-Glycoprotein Expression. Frontiers in Pharmacology. 2021; 12: 653306.

[22] Oba T, Izumi H, Ito K. ABCB1 and ABCC11 confer resistance to eribulin in breast cancer cell lines. Oncotarget. 2016; 7: 7001170027.

[23] Wei L, Wu Z, Yang Y, Zhao M, Ma X, Li J, et al. Overexpression of ABCG2 confers resistance to pevonedistat, an NAE inhibitor. Experimental Cell Research. 2020; 388: 111858. 
[24] Chen XY, Yang Y, Wang JQ, Wu ZX, Li J, Chen ZS. Overexpression of ABCC1 Confers Drug Resistance to Betulin. Frontiers in Oncology. 2021; 11: 640656.

[25] Wu Z, Mai Q, Yang Y, Wang J, Ma H, Zeng L, et al. Overexpression of human ATP-binding cassette transporter ABCG2 contributes to reducing the cytotoxicity of GSK1070916 in cancer cells. Biomedicine \& Pharmacotherapy. 2021; 136: 111223.

[26] Hyer ML, Milhollen MA, Ciavarri J, Fleming P, Traore T, Sappal $\mathrm{D}$, et al. A small-molecule inhibitor of the ubiquitin activating enzyme for cancer treatment. Nature Medicine. 2018; 24: 186193.

[27] Barghout SH, Patel PS, Wang X, Xu GW, Kavanagh S, Halgas $\mathrm{O}$, et al. Preclinical evaluation of the selective smallmolecule UBA1 inhibitor, TAK-243, in acute myeloid leukemia. Leukemia. 2019; 33: 37-51.

[28] Jin J, Li X, Gygi SP, Harper JW. Dual E1 activation systems for ubiquitin differentially regulate E2 enzyme charging. Nature. 2007; 447: 1135-1138.

[29] Swatek KN, Komander D. Ubiquitin modifications. Cell Research. 2016; 26: 399-422.

[30] Wertz IE, Wang X. From Discovery to Bedside: Targeting the Ubiquitin System. Cell Chemical Biology. 2019; 26: 156-177.

[31] Besse A, Stolze SC, Rasche L, Weinhold N, Morgan GJ, Kraus $\mathrm{M}$, et al. Carfilzomib resistance due to ABCB1/MDR1 overexpression is overcome by nelfinavir and lopinavir in multiple myeloma. Leukemia. 2018; 32: 391-401.

[32] Kathawala RJ, Espitia CM, Jones TM, Islam S, Gupta P, Zhang $\mathrm{YK}$, et al. ABCG2 Overexpression Contributes to Pevonedistat Resistance. Cancers. 2020; 12: 429.

[33] Tong Z, Yerramilli U, Surapaneni S, Kumar G. The interactions of lenalidomide with human uptake and efflux transporters and UDP-glucuronosyltransferase 1a1: lack of potential for drug-drug interactions. Cancer Chemotherapy and Pharmacology. 2014; 73: 869-874.

[34] Hartz AMS, Zhong Y, Shen AN, Abner EL, Bauer B. Preventing P-gp Ubiquitination Lowers A $\beta$ Brain Levels in an Alzheimer's Disease Mouse Model. Frontiers in Aging Neuroscience. 2018; 10: 186.

[35] Murai Y, Jo U, Murai J, Jenkins LM, Huang SN, Chakka S, et al. SLFN11 Inactivation Induces Proteotoxic Stress and Sensitizes Cancer Cells to Ubiquitin Activating Enzyme Inhibitor TAK243. Cancer Research. 2021; 81: 3067-3078.

[36] Zhuang J, Shirazi F, Singh RK, Kuiatse I, Wang H, Lee HC, et al. Ubiquitin-activating enzyme inhibition induces an unfolded protein response and overcomes drug resistance in myeloma. Blood. 2019; 133: 1572-1584.

[37] Barghout SH, Aman A, Nouri K, Blatman Z, Arevalo K, Thomas GE, et al. A genome-wide CRISPR/Cas9 screen in acute myeloid leukemia cells identifies regulators of TAK-243 sensitivity. JCI Insight. 2021; 6: e141518.

[38] Wu ZX, Yang Y, Wang JQ, Narayanan S, Lei ZN, Teng QX, et al. Overexpression of ABCG2 Confers Resistance to MLN7243, a Ubiquitin-Activating Enzyme (UAE) Inhibitor. Frontiers in Cell and Developmental Biology. 2021; 9: 697927.

[39] Akiyama S, Fojo A, Hanover JA, Pastan I, Gottesman MM. Isolation and genetic characterization of human KB cell lines resistant to multiple drugs. Somatic Cell and Molecular Genetics. 1985; 11: 117-126.

[40] Aoki S, Chen ZS, Higasiyama K, Setiawan A, Akiyama S, Kobayashi M. Reversing effect of agosterol a, a spongean sterol acetate, on multidrug resistance in human carcinoma cells. Japanese Journal of Cancer Research. 2001; 92: 886-895.

[41] Hu Y, Krishan A, Nie W, Sridhar KS, Mayer LD, Bally M. Synergistic cytotoxicity of pyrazoloacridine with doxorubicin, etoposide, and topotecan in drug-resistant tumor cells. Clinical Cancer Research. 2004; 10: 1160-1169.

[42] Wu ZX, Peng Z, Yang Y, Wang JQ, Teng QX, Lei ZN, et al. M3814, a DNA-PK Inhibitor, Modulates ABCG2-Mediated Multidrug Resistance in Lung Cancer Cells. Frontiers in Oncology. 2020; 10: 674.

[43] Wu ZX, Yang Y, Teng QX, Wang JQ, Lei ZN, Wang JQ, et al. Tivantinib, A c-Met Inhibitor in Clinical Trials, Is Susceptible to ABCG2-Mediated Drug Resistance. Cancers. 2020; 12: 186.

[44] Feng W, Zhang M, Wu ZX, Wang JQ, Dong XD, Yang Y, et al. Erdafitinib Antagonizes ABCB1-Mediated Multidrug Resistance in Cancer Cells. Frontiers in Oncology. 2020; 10: 955.

[45] Wang J, Yang DH, Yang Y, Wang JQ, Cai CY, Lei ZN, et al. Overexpression of ABCB1 Transporter Confers Resistance to mTOR Inhibitor WYE-354 in Cancer Cells. International Journal of Molecular Sciences. 2020; 21: 1387.

[46] Wang J, Wang B, Lei Z, Teng Q, Li JY, Zhang W, et al. Derivative of 5-cyano-6-phenylpyrimidin antagonizes ABCB1and ABCG2-mediated multidrug resistance. European Journal of Pharmacology. 2019; 863: 172611.

[47] Alam A, Kowal J, Broude E, Roninson I, Locher KP. Structural insight into substrate and inhibitor discrimination by human Pglycoprotein. Science. 2019; 363: 753-756.

[48] Trott O, Olson AJ. AutoDock Vina: Improving the speed and accuracy of docking with a new scoring function, efficient optimization, and multithreading. Journal of Computational Chemistry. 2009 ; 65: 455-461.

[49] Rolfe M. The Holy Grail: Solid Tumor Efficacy by Proteasome Inhibition. Cell Chemical Biology. 2017; 24: 125-126.

[50] Loscocco F, Visani G, Ruzzo A, Bagaloni I, Fuligni F, Galimberti $\mathrm{S}$, et al. Clinical Relevance of $\mathrm{ABCB} 1, \mathrm{ABCG} 2$, and ABCC2 Gene Polymorphisms in Chronic Myeloid Leukemia Patients Treated With Nilotinib. Frontiers in Oncology. 2021; 11: 672287.

[51] Eadie LN, Dang P, Saunders VA, Yeung DT, Osborn MP, Grigg $\mathrm{AP}$, et al. The clinical significance of ABCB1 overexpression in predicting outcome of CML patients undergoing first-line imatinib treatment. Leukemia. 2017; 31: 75-82.

[52] Brendel C, Scharenberg C, Dohse M, Robey RW, Bates SE, Shukla $\mathrm{S}$, et al. Imatinib mesylate and nilotinib (AMN107) exhibit high-affinity interaction with ABCG2 on primitive hematopoietic stem cells. Leukemia. 2007; 21: 1267-1275.

[53] Kunická T, Souček P. Importance of ABCC1 for cancer therapy and prognosis. Drug Metabolism Reviews. 2014; 46: 325-342.

[54] Vaidyanathan A, Sawers L, Gannon A, Chakravarty P, Scott AL, Bray SE, et al. ABCB1 (MDR1) induction defines a common resistance mechanism in paclitaxel- and olaparib-resistant ovarian cancer cells. British Journal of Cancer. 2016; 115: 431-441.

[55] Leggas M, Panetta JC, Zhuang Y, Schuetz JD, Johnston B, Bai F, et al. Gefitinib Modulates the Function of Multiple ATPBinding Cassette Transporters In vivo. Cancer Research. 2006; 66: 4802-44807.

[56] Mlejnek P, Kosztyu P, Dolezel P, Bates SE, Ruzickova E. Reversal of $\mathrm{ABCB} 1$ mediated efflux by imatinib and nilotinib in cells expressing various transporter levels. Chemico-Biological Interactions. 2017; 273: 171-179.

[57] Chufan EE, Kapoor K, Sim H, Singh S, Talele TT, Durell SR, et $a l$. Multiple transport-active binding sites are available for a single substrate on human P-glycoprotein (ABCB1). PLoS ONE. 2014; 8: e82463.

[58] Jackson SM, Manolaridis I, Kowal J, Zechner M, Taylor NMI, Bause M, et al. Structural basis of small-molecule inhibition of human multidrug transporter ABCG2. Nature Structural \& Molecular Biology. 2018; 25: 333-340. 